\title{
Unmixing the Galactic halo with RR Lyrae tagging
}

\author{
V. Belokurov,${ }^{1,2 \star}$ A. J. Deason, ${ }^{3 \star}$ S. E. Koposov, ${ }^{1,4}$ M. Catelan,,${ }^{5,6}$ D. Erkal, ${ }^{1,7}$ \\ A. J. Drake ${ }^{8}$ and N. W. Evans ${ }^{1}$ \\ ${ }^{1}$ Institute of Astronomy, Madingley Rd, Cambridge CB3 OHA, UK \\ ${ }^{2}$ Center for Computational Astrophysics, Flatiron Institute, 162 5th Avenue, New York, NY 10010, USA \\ ${ }^{3}$ Institute for Computational Cosmology, Department of Physics, University of Durham, South Road, Durham DH1 3LE, UK \\ ${ }^{4}$ Department of Physics, McWilliams Center for Cosmology, Carnegie Mellon University, 5000 Forbes Avenue, Pittsburgh, PA 15213, USA \\ ${ }^{5}$ Instituto de Astrofísica, Pontificia Universidad Católica de Chile, Av. Vicuña Mackenna 4860, $782-0436$ Macul, Santiago, Chile \\ ${ }^{6}$ Millennium Institute of Astrophysics, Santiago, Chile \\ ${ }^{7}$ Department of Physics, University of Surrey, Guildford GU2 7XH, UK \\ ${ }^{8}$ California Institute of Technology, 1200 E. California Blvd, CA 91225, USA
}

Accepted 2018 February 27. Received 2018 January 31; in original form 2017 November 20

\begin{abstract}
We show that tagging RR Lyrae stars according to their location in the period-amplitude diagram can be used to shed light on the genesis of the Galactic stellar halo. The mixture of RR Lyrae of ab type, separated into classes along the lines suggested by Oosterhoff, displays a strong and coherent evolution with Galactocentric radius. The change in the RR Lyrae composition appears to coincide with the break in the halo's radial density profile at $\sim 25 \mathrm{kpc}$. Using simple models of the stellar halo, we establish that at least three different types of accretion events are necessary to explain the observed RRab behaviour. Given that there exists a correlation between the RRab class fraction and the total stellar content of a dwarf satellite, we hypothesize that the field halo RRab composition is controlled by the mass of the progenitor contributing the bulk of the stellar debris at the given radius. This idea is tested against a suite of cosmological zoom-in simulations of Milky Way-like stellar halo formation. Finally, we study some of the most prominent stellar streams in the Milky Way halo and demonstrate that their RRab class fractions follow the trends established previously.
\end{abstract}

Key words: stars: variables: RR Lyrae-galaxies: dwarf-Local Group-galaxies: structure.

\section{INTRODUCTION}

'Chemical tagging' postulates the existence of a stellar fingerprint - a unique pattern of elemental abundances - that each star carries and that can be used to trace it back to its location of origin (Freeman \& Bland-Hawthorn 2002). This hypothesis has motivated many spectroscopic surveys (Allende Prieto et al. 2008; Cui et al. 2012; Gilmore et al. 2012; De Silva et al. 2015; Majewski et al. 2017) and has kick-started a number of observational (e.g. Helmi et al. 2006; Majewski et al. 2012; Blanco-Cuaresma et al. 2015) and theoretical (e.g. Font et al. 2006; Roškar et al. 2008; BlandHawthorn, Krumholz \& Freeman 2010) investigations, opening a new field of astrophysical enquiry - Galactic chemodynamics (see e.g. Schönrich \& Binney 2009; Minchev, Chiappini \& Martig 2013, 2014). The application of the 'chemical tagging' idea to the studies of the Galactic disc has enjoyed plenty of success (e.g. De Silva et al. 2007; Bensby, Feltzing \& Oey 2014; Bovy 2016), yet inevitably, has uncovered a number of stumbling blocks too (see e.g.

^E-mail: vasily@ast.cam.ac.uk (VB); alis.j.deason@durham.ac.uk (AJD)
Mitschang et al. 2014; Ting, Conroy \& Goodman 2015; Ness et al. 2018).

In the Milky Way's (MW's) halo, progress has so far been much slower, mainly because acquiring a large number of high-resolution spectra of stars across a range of halo locations remains prohibitively arduous. For example, the most recent attempt to survey the halo at high resolution beyond the solar neighbourhood (see Battaglia et al. 2017) includes only 28 stars, whose heliocentric distances are mostly limited to within $30 \mathrm{kpc}$. Note, however, that while the field halo remains poorly explored (in terms of precision chemistry), there exists a large amount of spectroscopic data on the surviving satellites. Curiously, while similar in concept, the genealogy of the disc and the halo differ substantially in practice. In the disc, chemodynamicists attempt to rewind the stars back to the low-mass star clusters they were born in. In the halo, a much richer variety of progenitors are available: stellar systems with masses from that of a giant molecular cloud to that of the Large Magellanic Cloud (LMC) could have all contributed to the halo formation.

For example, by comparing the abundance trends in the globular clusters (GCs) and the local halo stars, several authors claim that as 
much as 50 per cent of the stellar halo could have originated in GCs (Carretta et al. 2010; Martell \& Grebel 2010; Martell et al. 2011). The above calculation relies heavily on the theory that the GCs were 10-20 times more massive in their youth and have experienced prolific mass loss since - the argument put in place to explain the ratios between the second and first generations of their member stars (see Gratton, Carretta \& Bragaglia 2012, for a review). However, the study of Deason, Belokurov \& Weisz (2015) argues against the stellar halo creation through GC disruption. They base their argument on the measurements of the ratio of the number of blue horizontal branch stars to that of blue stragglers. Bear in mind though that, as Chung, Lee \& Pasquato (2016) show, this constraint may perhaps be circumvented by invoking the loss of most of the first generation stars early in a cluster's life.

At the other end of the mass spectrum, observational evidence is mounting for the most massive dwarf galaxies to contribute a significant fraction of the stellar halo. First, according to the $\alpha$-elements to iron abundance ratio (as gleaned both from low- and high-resolution spectroscopy), the local stellar halo appears similar to galaxies like the LMC and Sgr (see e.g. Venn et al. 2004; Tolstoy, Hill \& Tosi 2009; de Boer et al. 2014). Additionally, the MW halo's radial density profile shows a break at around $25 \mathrm{kpc}$ (Watkins et al. 2009; Deason, Belokurov \& Evans 2011; Sesar, Jurić \& Ivezić 2011), which according to Deason et al. (2013) could be best explained with an early accretion of a massive stellar system. The final clue can be found in the study of the make-up of the RR Lyrae population of the Galactic halo. For example, Stetson et al. (2014) and Fiorentino et al. (2015) demonstrate that high-amplitude short-period (HASP) RRab stars can be used to decipher the relative contributions of the GCs, ultrafaint dwarfs (UFDs), classical dwarf spheroidals (dSphs), and massive systems such as the LMC and the Small Magellanic Cloud (SMC). They point out that while the HASP RR Lyrae are a common denizen of the halo, these stars are completely lacking in most surviving dwarfs (including all UFDs), except for Sagittarius (Sgr) and the Magellanic Clouds. In GCs, as Fiorentino et al. (2015) show, only the more metal-rich systems have sizeable HASP populations. Thus the only pathway to HASP creation is via a massive system with rapid metal enrichment. This picture is in full agreement with the earlier analysis of the period-amplitude distribution (known as the Bailey diagram) of the RR Lyraes in the halo and the MW satellites (see e.g. Catelan 2009; Zinn et al. 2014). Note, in addition, an interesting mechanism for the HASP pulsator production via hysteresis-like evolution of the RR Lyrae along the horizontal branch, as presented in Bono et al. (1997).

The hypothesis of the stellar halo creation by way of a massive dwarf galaxy disruption does not directly contradict the evidence for a substantial GC contribution. Incontrovertibly, GCs do not form on their own but always require a host, an unlucky galaxy destined to be tidally destroyed (see e.g. Kruijssen 2015; Bekki \& Tsujimoto 2016; Boylan-Kolchin 2017; Renaud, Agertz \& Gieles 2017). Note that these models also allow formation of some of the metal-rich clusters in situ (also see formation scenarios discussed in Carretta et al. 2010). However, it seems likely that an in situ stellar halo would possess some residual spin still observable at the present day. Indeed, in the MW, there have been some claims of a detection of an in situ halo population (see e.g. Carollo et al. 2007, 2010). However, given that no substantial rotation has been reported for the Galactic metal-poor halo tracers (see Deason et al. 2017), it is safe to assume that the in situ contribution to the MW old halo at high Galactic $|z|$ is minimal.

Importantly, given that the debris mixing times are a strong function of Galactocentric radius and that the dynamical friction depends most notably on the satellite's mass and its orbital parameters, it is naive to expect the properties of the stellar halo to be the same across the Galaxy. Unfortunately, the exploration of the evolution in the stellar halo's make-up has been hindered by the excessive cost of running a spectroscopic survey of such an enormous volume at such a low target density. In this paper, we propose to decipher the fractional contributions of stellar systems of different masses by mapping out the change in the mixture of pulsating RR Lyrae variables.

Our proposed tagging scheme relies on the ideas of Oosterhoff $(1939,1944)$, who pointed out striking differences in the period distributions of the fundamental-mode (i.e. RRab) stars in GCs. Specifically, clusters in the MW halo appear to be separable into two classes (the 'Oosterhoff dichotomy'), one with a mean period $\left\langle P_{\mathrm{ab}}\right\rangle \approx 0.55 \mathrm{~d}$ and another with $\left\langle P_{\mathrm{ab}}\right\rangle \approx 0.65 \mathrm{~d}-$ nowadays commonly referred to as groups Oosterhoff I (OoI) and Oosterhoff II (OoII), respectively. In between the two, one finds the so-called 'Oosterhoff gap', with few bona fide MW globulars (the 'Oosterhoff-intermediate' [OoInt] ones) being present over the range $0.58 \lesssim\left\langle P_{\mathrm{ab}}\right\rangle \lesssim 0.62 \mathrm{~d}$ (Catelan 2004, 2009). Arp (1955) and Preston (1959) showed early on that there is a tendency for OoII clusters to be more metal-poor than those of type OoI, while more recently, it has also been found that OoII objects are slightly older than OoI systems, on average (e.g. Gratton et al. 2010; Sollima et al. 2014). OoII clusters have characteristically bluer horizontal branches (HBs) than do OoI clusters. The RR Lyrae are also brighter in OoII clusters, possibly due to a combination of evolutionary effects (e.g. van Albada \& Baker 1973; Lee, Demarque \& Zinn 1990; Pritzl et al. 2002; Sollima et al. 2014) and an increased He abundance, compared to the RR Lyrae in OoI systems (e.g. Gratton et al. 2010; Jang et al. 2014; Jang \& Lee 2015; VandenBerg, Denissenkov \& Catelan 2016, and references therein). As discussed by Sollima et al. (2014) and VandenBerg et al. (2016), none of the proposed solutions account for all the available data, and it can thus be concluded that a fully satisfactory explanation of the Oosterhoff dichotomy has not yet been achieved. What does appear clear, however, is that the lack of OoInt GCs is due to the non-monotonic behaviour of $\mathrm{HB}$ morphology with metallicity: the metallicity range $-2.0 \lesssim[\mathrm{Fe} / \mathrm{H}] \lesssim-1.8$ is largely occupied by GCs with completely blue HBs, which have accordingly failed to produce RR Lyrae stars in significant numbers (Castellani 1983; Renzini 1983). The reason for this non-monotonic behaviour is not fully understood, but could be a consequence of the way mass-loss efficiency on the red giant branch changes as a function of metallicity (e.g. Dotter 2008, and references therein).

The fact that the Oosterhoff phenomenon carries important information to decipher the earliest stages of the formation of the MW halo is confirmed not only by its dependence on metallicity and age as discussed above, but also by the presence of correlations between Oosterhoff status and the spatial position and orbital properties of RR Lyrae-bearing systems (e.g. van den Bergh 1993c, 2011; Lee \& Carney 1999). Note, in addition, that while OoInt GCs are not present in large numbers in the MW halo, the situation changes dramatically in the immediate vicinity of our Galaxy, where it has been shown that the OoInt region is actually favoured, compared with the OoI and OoII loci, in $\left\langle P_{\mathrm{ab}}\right\rangle-[\mathrm{Fe} / \mathrm{H}]$ and other similar diagrams. This suggests that the MW halo cannot simply have formed from the accretion of the early counterparts of the present-day population of MW dwarf satellites (Catelan 2004, 2009; Stetson et al. 2014). On the other hand, most of the UFDs have been found to be OoII systems, which is consistent with their very low metallicities and the trends seen in the MW halo (e.g. Clementini 2014; Vivas et al. 
2016) - though it should be noted that some of the more RR Lyraerich UFD systems, such as CVn I and UMa I, like most of their more massive dSph counterparts, are actually OoInt (Kuehn et al. 2008; Garofalo et al. 2013).

Presented with the distinct behaviour of RR Lyrae in the periodamplitude space as described above, we put forward a simple observational diagnostic for the genesis of the Galactic field halo population. We propose to shed light on to the likely halo progenitors by mapping out the fraction of the pulsators occupying different locations in the Bailey diagram. This analysis is contingent on the fact that the MW satellites do not contain RRab stars of a particular Oosterhoff type, but rather host mixtures of these (Catelan 2009). Here, we concentrate in particular on the difference in the 3D distribution of the RRab stars that fall approximately into the OoI and OoII groups (see Section 2 for details). Moreover, motivated by the results presented in Stetson et al. (2014) and Fiorentino et al. (2015), we compare the behaviour of these two classes with the spatial evolution of the fraction of the HASP variables. Note that, compared to the multitude of in-depth studies of the Oosterhoff dichotomy in the surviving satellites of the Galaxy, little has been done so far with regards to the field RR Lyrae population. This is not an omission but rather a delay due to the lack (until recently) of large all-sky RR Lyrae samples.

While precious few in number, several previous studies of the period-amplitude make-up of the MW field population exists. For example, the Oosterhoff dichotomy in the Galactic halo RRab stars is studied in Miceli et al. (2008), who find that within $20-30 \mathrm{kpc}$ from the Galactic Centre, the OoII RRabs follow a steeper radial density law compared to those belonging to the OoI group. Zinn et al. (2014) explore not only the field halo but also the prominent halo substructures crossing the field-of-view of their $\sim 800 \mathrm{deg}^{2}$ survey, such as the Sgr stream and the Virgo stellar stream (VSS). They measure the shape of the stellar halo and see a clear break (see the discussion above) in the radial density law at $\sim 25 \mathrm{kpc}$. While Zinn et al. (2014) detect no noticeable change in the Oosterhoff mixture across the break, they caution that this could simply be due to the small RR Lyrae sample size available to them. Concentrating on the Sgr stream, they notice that the stream contains a smaller fraction of short-period pulsators (compared to the remnant) and link this to the chemical abundance gradients in the progenitor. Most importantly, they point out a great level of similarity between the period-amplitude distribution in the field halo and in the large substructures such as the Sgr stream and the VSS. Moving closer to the centre of the Galaxy, Kunder \& Chaboyer (2009) scrutinize the bulge RR Lyrae population and notice an apparent difference between the periodamplitude distribution of the bulge RR Lyrae and of those elsewhere in the MW. More precisely, they observe a much higher fraction of shorter period objects (at fixed amplitude), which they link to an enhanced metal enrichment (also see Kunder et al. 2013; Pietrukowicz et al. 2015, for an updated analysis). Based on the observed difference, Kunder \& Chaboyer (2009) conjecture that the progenitor(s) of the Galactic bulge ought to be distinct from the halo parent system(s).

This paper is structured as follows. In Section 2, we describe the sources of the RR Lyrae data used here and the selection boundaries in the period-amplitude space. We show how the mixture of RRab stars from different portions of the Bailey diagram evolves with Galactocentric radius in Section 3. The Oosterhoff dichotomy in the currently known most prominent stellar streams is discussed in Section 4. Finally, we provide the summary and the context for this study in Section 5.

\section{RR LYRAE DATA}

This work relies, in part, on the all-sky RR Lyrae data recently made publicly available by the Catalina Real-Time Transient Survey (CRTS). The CRTS sample was released in several instalments: the two largest subsets, namely the northern sky as reported in Drake et al. (2013a) and the complementary southern portion analysed in Torrealba et al. (2015), contain the bulk of the data. To these we add the RR Lyrae from Drake et al. (2013b, 2014, 2017). After the crossmatch of all five catalogues, 31301 unique RR Lyrae survive. The distribution of their periods and amplitudes is shown in the left-hand panel of Fig. 1. Note that, in this analysis, the CRTS amplitudes are corrected by 0.15 mag as explained in Drake et al. (2013a). Here, we use the RR Lyrae heliocentric distances as published in the above catalogues. To convert to Galactocentric distances, we assume a solar position $8 \mathrm{kpc}$ away from the Galactic Centre. Only RRab stars are considered in this work. These are selected according to the following period $(P)$ and amplitude (Amp) criteria:

$$
\begin{array}{r}
0.43<P(\mathrm{~d})<0.9, \\
0.6<\operatorname{Amp}(\mathrm{mag})<1.45, \\
\text { Amp }<3.75-3.5 P .
\end{array}
$$

This first cut ensures that, compared to the bulk of the RRab stars, possible contaminating variables with shorter (a minuscule contamination from RRc stars at short periods and small amplitude is still possible) or longer periods are excluded. Note that we have checked that the results presented below do not change significantly if the lower amplitude boundary is increased. The second cut attempts to minimize the effects of the CRTS selection efficiency related to the pulsation amplitude. Only stars with Amp $>0.6$ mag are detected out to $50 \mathrm{kpc}$ without a significant loss in completeness. This is demonstrated in the right-hand panel of Fig. 1, where the density of the CRTS RR Lyrae in the space spanned by the amplitude and the Galactocentric distance is displayed. Indeed, the low-amplitude stars are only detected at small distances. However - as emphasized by the black horizontal line - no strong distancedependent selection bias is visible in this figure for the RR Lyrae with Amp $>0.6$ mag located within $50 \mathrm{kpc}$ from the Galactic Centre. There are $N_{\text {tot }}=27491$ RRab stars available after this selection. The OoI and OoII stars are demarcated by the line given in equation (11) of Zorotovic et al. (2010) but offset by +0.15 in amplitude, namely

Amp $=-2.477-22.046 \log P-30.876(\log P)^{2}$.

As evidenced by Fig. 1, this selection boundary wraps tightly around the overdensity of OoI stars. Note that, according to the above definition, the Class 2 will include both OoII and OoInt objects. From here onwards, we refer to the RRab stars falling within these selection boxes as Type (or Class) 1 and 2 stars. The inset in the lower panel of Fig. 1 shows the amplitude distributions of the RR Lyrae belonging to the two types. The two histograms appear barely distinguishable, thus supporting our assumption of the likely constancy of the selection effects with Class membership.

Finally, we select HASP RR Lyrae according to the following conditions:

$$
0.43<P(\mathrm{~d})<0.48,
$$

$0.9<\operatorname{Amp}(\mathrm{mag})<1.45$.

We complement the CRTS data with a sample of RR Lyrae stars in GC and dSph satellites of the MW. The GC RR Lyrae are taken from the updated version (see Clement 2017a,b) of the catalogue presented by Clement et al. (2001). Finally, we also use the following catalogues of RR Lyrae stars in the MW dSphs: in Cetus and Tucana by Bernard et al. (2009), in Draco by Kinemuchi et al. 

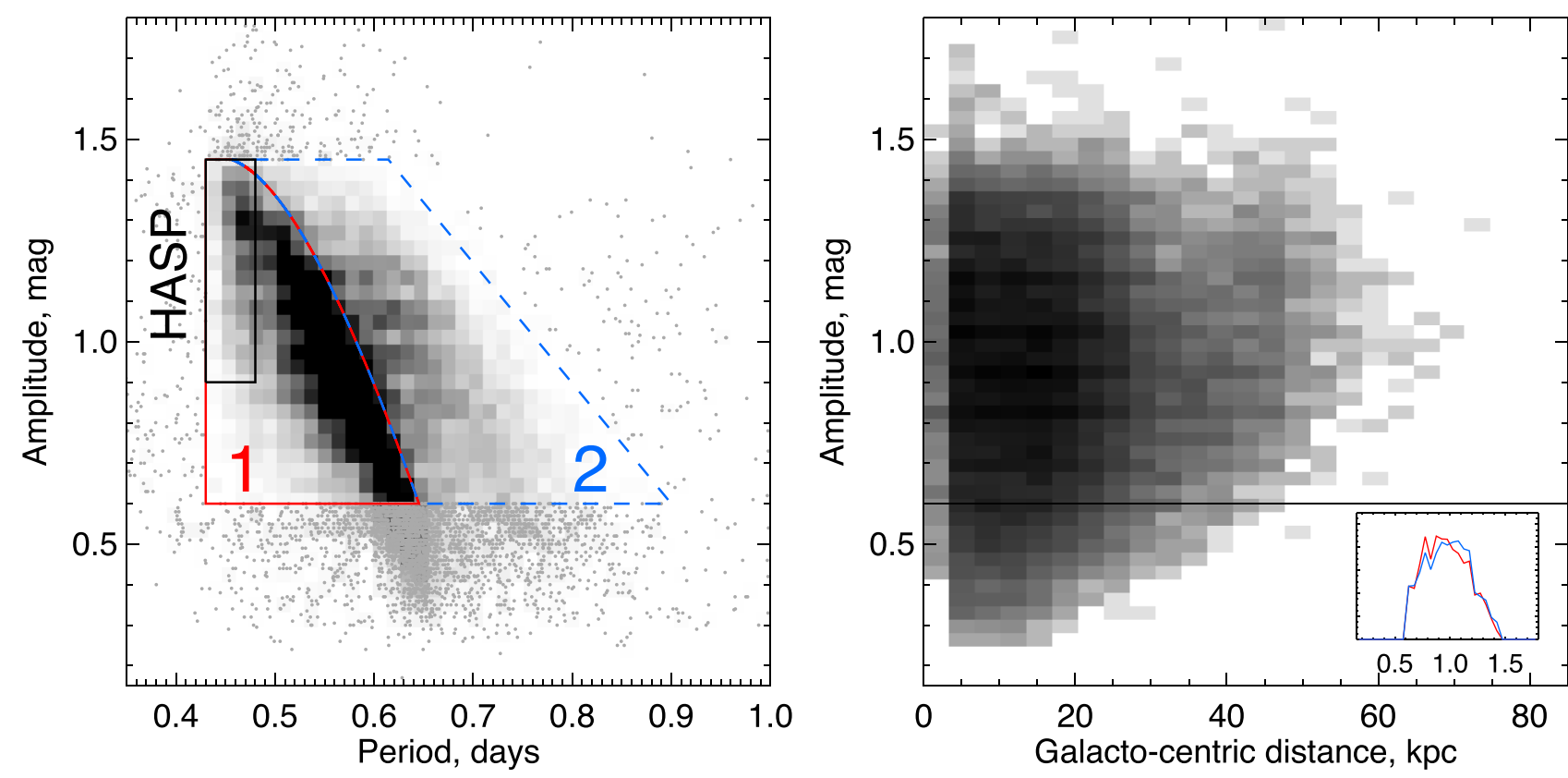

Figure 1. Left: density of CRTS RR Lyrae stars in the plane of $V$-band amplitude (in magnitudes) and period (in days). Locations of the Type 1 (red), Type 2 (blue), and the HASP (black) objects are shown. Selection boundaries are stipulated in the main text. Note that according to this selection, Type 1 objects are predominantly OoI RR Lyrae, while Type 2 is composed of OoII and OoInt objects. Right: logarithm of the CRTS RR Lyrae density in the space spanned by the amplitude and Galactocentric distance. As the shape of the lower edge of the distribution indicates, the completeness of the low-amplitude objects is a strong function of distance. However, for stars with Amp $>0.6 \mathrm{mag}$ (black horizontal line), the effects of the selection bias appear to be minimal. The inset shows the histograms of the amplitude for the stars in Class 1 (red) and Class 2 (blue). Given that the two distributions are nearly identical, we assume that the completeness affects both classes equally.

(2008), in Carina by Coppola et al. (2013), in Fornax by Greco et al. (2009), in Leo I by Stetson et al. (2014), in Sculptor by Kaluzny et al. (1995), in the LMC by Soszynski et al. (2003), and in the SMC by Kapakos, Hatzidimitriou \& Soszyński (2011). For the Sgr dwarf, we use the catalogue of Soszyński et al. (2014) and extract all RR Lyrae within $10^{\circ}$ from the centre of the dwarf and located between 22 and $31 \mathrm{kpc}$ from the Sun. We also use RR Lyrae in some of the brightest UFDs, such as Boo I (Dall'Ora et al. 2006), UMa I (Garofalo et al. 2013), and CVn I (Kuehn et al. 2008).

\section{RRAB MIXTURE EVOLUTION WITH GALACTOCENTRIC RADIUS}

Fig. 2 displays the change in the fraction of Type 1 (red) RR Lyrae with respect to all stars selected using equations (1), e.g. $f_{1}(R)=N_{1}(R) / N_{\text {tot }}(R)$, as a function of Galactocentric radius. By design, $N_{\text {tot }}=N_{1}+N_{2}$, thus $f_{2}=1-f_{1}$. All type fractions come with their associated uncertainties, which are computed by propagating the Poisson errors. As we are not taking the effects of the completeness into account, the above errors should be considered as lower bounds. Note that for this and other figures showing the change in the RR Lyrae mixture with radius, we apply additional cuts in Galactic height $z$ (to minimize the contamination form the thick disc) and extinction:

$$
\begin{array}{r}
E(B-V)<0.25, \\
|z|>1 \mathrm{kpc} .
\end{array}
$$

For each star, the reddening is obtained using the maps of Schlegel, Finkbeiner \& Davis (1998). We also exclude the most significant halo substructure, i.e. the Sgr stream (see figure caption for details). After the three extra cuts, we are left with $N_{\text {tot }}=19669$ RRab stars, of these 13267 are Type 1, 6402 are Type 2, and 1538 are HASP.
Also shown in Fig. 2 is the fraction of Type 2 (HASP) objects in blue (black). While the CRTS completeness is a strong function of magnitude and to a lesser extent of position on the sky, we believe that the selection biases affect in equal measure the stars in the three groups considered. This, of course, assumes that there are no dramatic differences in the amplitude distributions of the two classes. This assumption appears to be valid given the amplitude distributions of the Classes 1 and 2 shown in the inset of the right-hand panel of Fig. 1. Thus, based on our experiments with the sample selection boundaries, we believe that the fraction curves displayed indicate the actual change in the RR Lyrae mixture throughout the Galaxy. Across the distance range allowed by the data $(5-50 \mathrm{kpc})$, the Type 1 objects dominate, comprising approximately two-thirds of the overall RR Lyrae population, in accordance with previous studies (see Miceli et al. 2008; Abbas et al. 2014; Zinn et al. 2014). As the figure evidently demonstrates, this fraction also varies significantly with radius: $f_{1}$ drops in the innermost halo, and also beyond $30 \mathrm{kpc}$. At the edge of the CRTS survey, the $f_{1}$ fraction drops to 50 per cent and most likely continues to decrease further in the outer halo of the Galaxy. The peak in $f_{1}$ fraction, somewhere between 20 and $30 \mathrm{kpc}$, appears to match the location of the break in the stellar halo's radial density profile (see Watkins et al. 2009; Deason et al. 2011; Sesar et al. 2011).

Evidently more dramatic is the change in the HASP fraction. The proportion of the HASP RR Lyrae changes by a factor of $>3$ from 10 percent around the Galactic Centre to 3 percent in the outer halo. Intriguingly, the $f_{\text {HASP }}$ behaviour does not match fully the evolution of $f_{1}$. First, similar to Type 1 objects, the proportion of HASP RR Lyrae drops around $30 \mathrm{kpc}$. However, in the inner parts of the Galaxy $R<10 \mathrm{kpc}, f_{\text {HASP }}$ shows a strong increase, in the range where $f_{1}$ is shown to be declining. Given that, by construction, the HASP subsample contains the RR Lyrae with the highest amplitude 


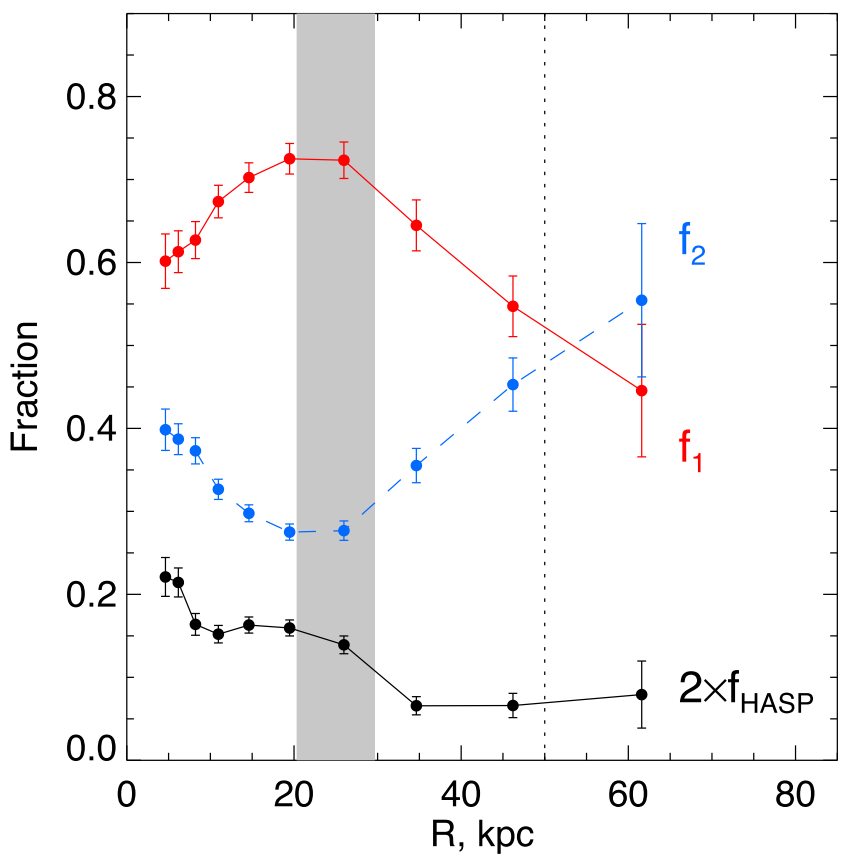

Figure 2. The RRab mixture evolution as a function of Galactocentric radius (kpc). Fractions $f_{1}$ (red), $f_{2}$ (blue), and $f_{\text {HASP }}$ (black, scaled up by a factor of 2) are each shown to vary significantly with radius. Note that $f_{\text {HASP }}+f_{1}+f_{2} \neq 1$ as HASP RRab are (almost entirely) a subset of the Type 1 objects. The red and blue curves are mirror images of each other by definition, whereas the HASP fraction $f_{\text {HASP }}$ behaves differently from both $f_{1}$ and $f_{2}$. The thick grey vertical line marks the location of a break in the stellar halo radial density profile (see Watkins et al. 2009; Deason et al. 2011; Sesar et al. 2011). The stars belonging to the Sgr stream (see Section 4) have been excluded by excising all objects with stream latitude $|B|<8^{\circ}$, where the stream coordinates are obtained by rotating RA and Dec. to align with the great circle with a pole at $(\alpha, \delta)=\left(303.63,59^{\circ} .68\right)$.

available, we believe that the measurement of the evolution of $f_{\text {HASP }}$ with radius is not significantly affected by the possible selection biases discussed earlier.

To illustrate the differences in the behaviour of Type 1/Type 2/HASP RR Lyrae with radius, Fig. 3 presents simple toy models of the fraction evolution: a ratio of power-law (Plummer) density profiles in dashed (dotted) black curves. Here, the Plummer density model is $n=n_{0}\left(1+r^{2} r_{\mathrm{s}}^{-2}\right)^{-5 / 2}$ with two parameters, describing the density normalization $n_{0}$ and the scale radius $r_{\mathrm{s}}$. The power law is simply $n=n_{0} r^{\alpha}$, where $\alpha$ is the power-law index. For the power-law density, we use the indices measured by Miceli et al. (2008), and given in their equations (28) and (29), namely, -2.7 for Class 1 and -3.2 for Class 2 . The density profiles suggested by Miceli et al. (2008) appear to give a reasonable description of the $f_{1}$ behaviour within the distance range probed by their data. Beyond $30 \mathrm{kpc}$, however, this model does not agree with the CRTS data: it predicts a continuing increase in the fraction of Type 1 stars, while a clear drop is registered. Using a ratio of two Plummer models with scale radii $30 \mathrm{kpc}(21 \mathrm{kpc})$ for Class 1 (2) achieves a marginal improvement, in particular in the very inner portions of the halo, from 5 to $15 \mathrm{kpc}$.

Given the $f_{1}$ behaviour out to $30 \mathrm{kpc}-$ as shown in Figs 2 and 3 one simple explanation of the change in the Galactic RRab mixture is to invoke two types of progenitors. In this scenario, a stellar system with a higher fraction of Type 1 RR Lyrae (Component 1) deposits tidal debris that then relaxes into a distribution with a flatter density profile as compared to the stars left behind by the progenitor(s)

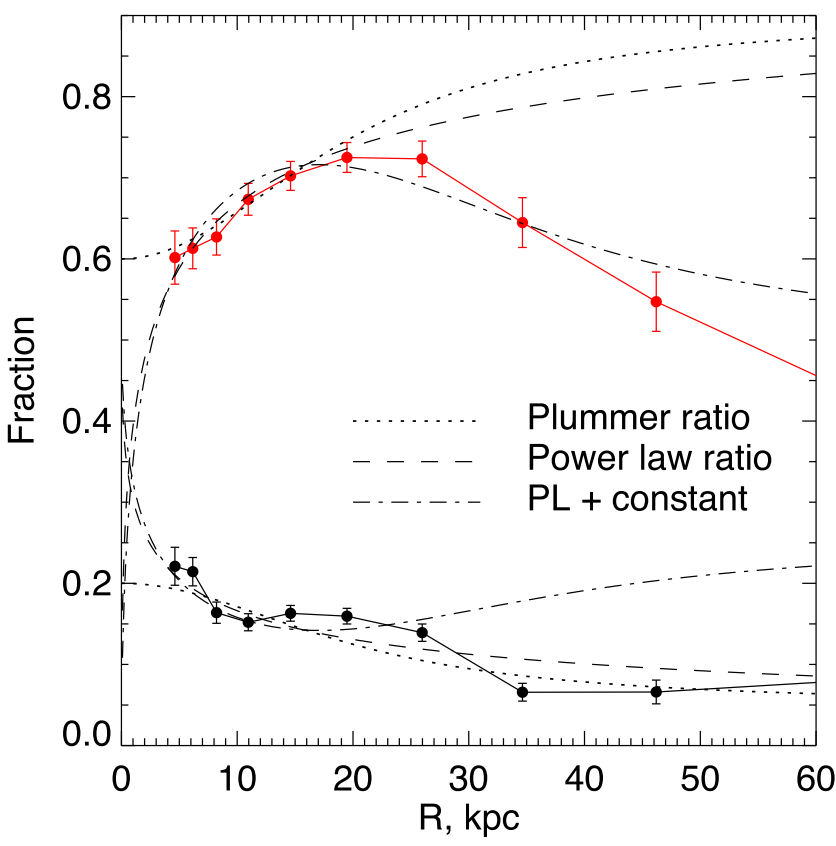

Figure 3. $f_{1}$ and $f_{\text {HASP }}$ radial profiles (as shown in Fig. 2) with toy mixture models overlaid. The dashed line shows the $f_{1}$ fraction in the halo where Type 1 and Type 2 RR Lyrae are distributed according to (spherical) powerlaw models reported in Miceli et al. (2008), namely: power-law index -2.7 for Type 1 and -3.2 for Type 2 . The dash-dotted line corresponds to a power-law density model as above but with an additional constant density component. The dotted line is a halo where each RRab type is represented with a Plummer density model: Type 1 with a scale radius of $30 \mathrm{kpc}$, and Type 2 with $21 \mathrm{kpc}$.

with a higher fraction of Type 2 RRab stars (Component 2). Please note that the models shown in Fig. 3 assume an extreme case where one type of progenitor contributes all RR Lyrae of a particular type. However, from our experiments with these simple models, it is clear that more realistic values $f_{1} \sim 0.2$ would also hold well against the data. This simple picture gives a convincing explanation of the fractional increase in Type 2 objects at small distances, but seemingly breaks down at large Galactocentric distances, where $f_{1}$ is observed to drop again.

Such a drop in the $f_{1}$ fraction (or, equivalently, increase in $f_{2}$ ) beyond the break radius could possibly be accounted for with an addition of a third progenitor type - that with an extremely flat radial density distribution, as illustrated by the dash-dotted line in Fig. 3. This model has an additional component whose density is constant with radius. As evident in the figure, the contribution of the third component appears to be enough to explain the turnover in the $f_{1}$ curve beyond $30 \mathrm{kpc}$. There is perhaps a more prosaic explanation for the peak and the turnover of the $f_{1}$ curve. Rather than requiring an additional component, such behaviour could probably be the result of the difference in sphericity between the Components 1 and 2 debris. The change in the stellar halo flattening with Galactocentric radius has recently been reported in a number of studies (see Xue et al. 2015; Das, Williams \& Binney 2016; Iorio et al. 2018). Quite simply, a significant vertical (with respect to the Galactic disc) flattening of the Component 1 debris would likely result in a decrease of $f_{1}$ at large radii. Finally, if, as argued by Deason et al. (2013), the break in the stellar halo density is created by the accretion of one massive stellar system, Component 1 might not be extending much beyond the apocentre of the host satellite, thus yielding a sharp truncation at around the break and subsequent drop in $f_{1}$. This 

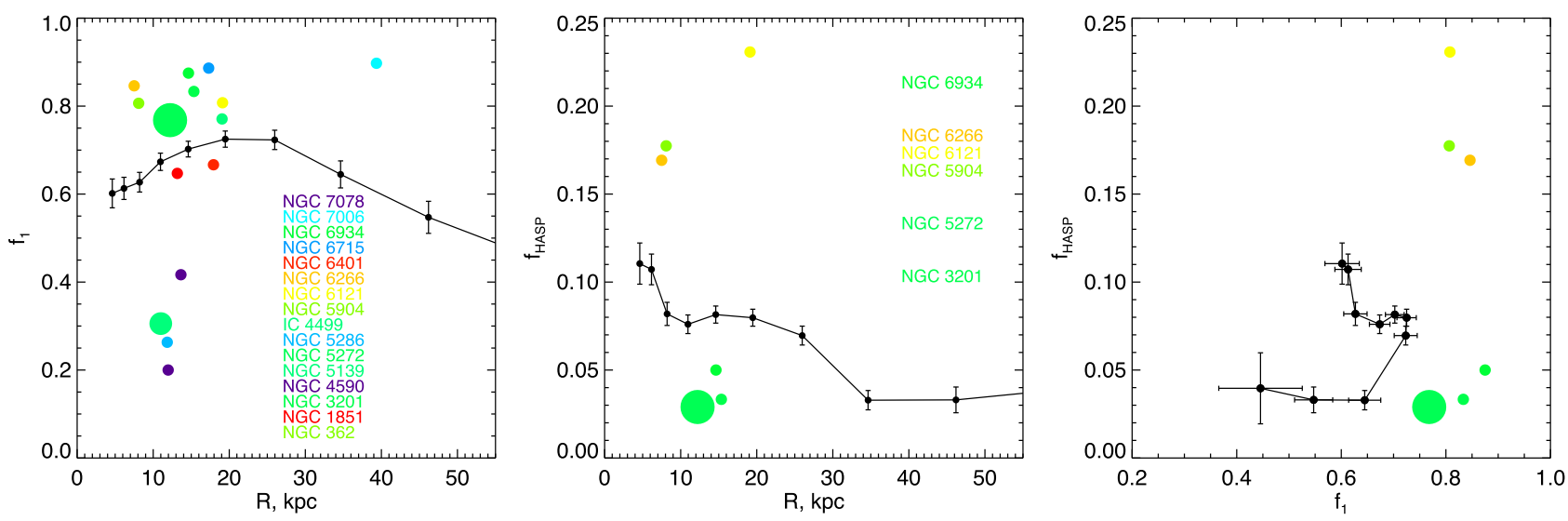

Figure 4. Left: fraction of Class 1 RRab stars in the MW GCs. The size of the circle represents the number of RR Lyrae in the cluster (with the smallest symbol corresponding to samples with $\leq 30$ objects and the largest to samples with as many as $\sim 140$ RRab stars), while the colour encodes the GC's metallicity, with violet/blue being the most metal-poor and orange/red the most metal-rich. Note the well-known Oosterhoff dichotomy where GCs tend to avoid intermediate values of $0.4<f_{1}<0.7$. Middle: fraction of HASP RRab stars $f_{\text {HASP }}$ in GCs. Very few, typically moderately metal-rich $([\mathrm{Fe} / \mathrm{H}]>-1.5$ dex), objects achieve $f_{\text {HASP }}>0.15$. Note that only objects with more than 1 HASP star are included in this panel. Right: the distribution of GCs in the space spanned by $f_{1}$ and $f_{\text {HASP. }}$ In all three panels, the black solid line shows the measurement of the Galactic stellar halo.

scenario, of course, works very well in conjunction with the previous hypothesis, i.e. that of a difference in the component flattening.

The evolution of the fraction of Type 1 RRab stars can be contrasted with the change in the fraction of HASP pulsators, shown as a black solid line in Figs 2 and 3. The HASP fraction evolves in the opposite sense to $f_{1}$ and out to $\sim 15 \mathrm{kpc}$ follows the trend in $f_{2}$ fraction. Beyond that, it switches from following $f_{2}$ and starts to track $f_{1}$. Apart from a bump in the $f_{\text {HASP }}$ profile at $\sim 20 \mathrm{kpc}$, it continues to fall precipitously as one moves into the outer halo. In summary, given this distinct evolution, changes in $f_{\mathrm{HASP}}$ cannot be described by the simple two-component model proposed above. Nonetheless, to illustrate the differences and commonalities between $f_{1}, f_{2}$, and $f_{\text {HASP }}$, Fig. 3 shows the inverted versions of the power law and the Plummer models described above. Clearly, both power-law density ratio and Plummer density ratio can explain crudely the global shape of the $f_{\text {HASP }}$ curve. For example, a simple three-component model can match the rise at the halo break radius, but does not have the subsequent fall-off in the outer halo.

\subsection{RRab mixture in Galactic satellites}

It is instructive to compare the mixture of the field halo RRab with that found in Galactic satellites, such as GCs and dSph galaxies. Fig. 4 compares $f_{1}$ (left-hand panel) and $f_{\text {HASP }}$ (middle panel) curves for the field halo (as described above) with the corresponding fractions in the GCs at the appropriate distance. The colour of the filled circle indicates the metallicity of the cluster, with blue (red) corresponding to relatively low (high) values of $[\mathrm{Fe} / \mathrm{H}]$. The size of the circle is proportional to the number of RR Lyrae in the cluster. As described in a number of previous studies, the GCs display a clear Oosterhoff dichotomy: $f_{1}$ fractions are clustered around $\sim 0.3$ and $\sim 0.8$, with few examples of intermediate values. The halo, on the other hand, occupies exactly the range avoided by the GCs, i.e. $0.5<f_{1}<0.75$. Unfortunately, there are not many RR Lyrae-rich GCs in the outer halo - most have extreme HB morphologies, either too red or too blue to produce significant numbers of RR Lyrae. Thus, it is not possible to track the behaviour of cluster $f_{1}$ with Galactocentric radius, with the majority of the data points lying within $20 \mathrm{kpc}$. With regard to $f_{\mathrm{HASP}}$, most GCs do not host as many HASP RRab stars as does the field halo at this radius. For a small number of clusters that have more than 1 HASP star, a dichotomy similar to that of Type $1 / 2$ is observed. Note, however, that the cluster data agree well with the outer halo $f_{\text {HASP }}$ fraction. Finally, the right-hand panel of Fig. 4 gives the track of the field halo in the space spanned by $f_{1}$ and $f_{\text {HASP }}$. Once again, this plot emphasizes the lack of GCs with intermediate $(\sim 0.6)$ values of $f_{1}$ and (simultaneously) high $(\sim 0.1)$ values of $f_{\text {HASP }}$. Note, however, that even though $f_{\text {HASP }}$ cannot be reliably measured for some of the GCs with low $f_{1}$ values, it is plausible that they contain sufficiently small (if any) number of these RR Lyrae. Therefore, with the current data, it is impossible to unambiguously rule out that a combination of disrupted GCs cannot yield a mixture of RR Lyrae similar to that of the field halo.

As pointed out in many previous studies, the $f_{1}$ fraction in dSphs, as shown in Fig. 5, is intermediate between those of GCs of types OoI and OoII. This is the well-known flip-side of the Oosterhoff dichotomy (see Section 1). On the other hand, the figure also shows that the values of $f_{1}$ seen amongst dSphs actually match those seen in the field halo. In and of itself, this does not necessarily imply that the halo field (or even individual $\mathrm{dSphs}$, for that matter) does not show the Oosterhoff dichotomy, as $f_{1}$ values that are intermediate between those seen in OoI and OoII objects could in principle also arise simply from an arbitrary mix containing these two types of stars. Note that, in the left- and right-hand panels, the dSph fractions are not shown at the satellites' distance but instead are grouped around the fiducial location of $20 \mathrm{kpc}$ (given that the bulk of the surviving dwarfs is beyond the reach of the CRTS). The colour of the symbol indicates the total stellar mass of the satellite, with low-mass systems (like Draco and Carina) shown in purple and blue and the largest galaxies (such as the Sgr dwarf and the Clouds) shown in orange and red. A clear correlation between the stellar mass and the $f_{1}$ fraction is visible (hints of this correlation are discussed in e.g. Stetson et al. 2014; Fiorentino et al. 2015). Note that only the most massive systems host enough Type 1 RRab stars (with the exception of Draco) to match the peak in the field halo at $f_{1} \sim 0.75$ at $\sim 25 \mathrm{kpc}$. Also shown as a large filled black circle is an estimate of the $f_{1}$ fraction for the brightest of the UFDs, namely Boo I, CVn I, and UMa I. While the latter two objects have values $f_{1} \sim 0.2$, already for Boo I this fraction is consistent with zero. Even though it does not seem possible to estimate $f_{1}$ in most 

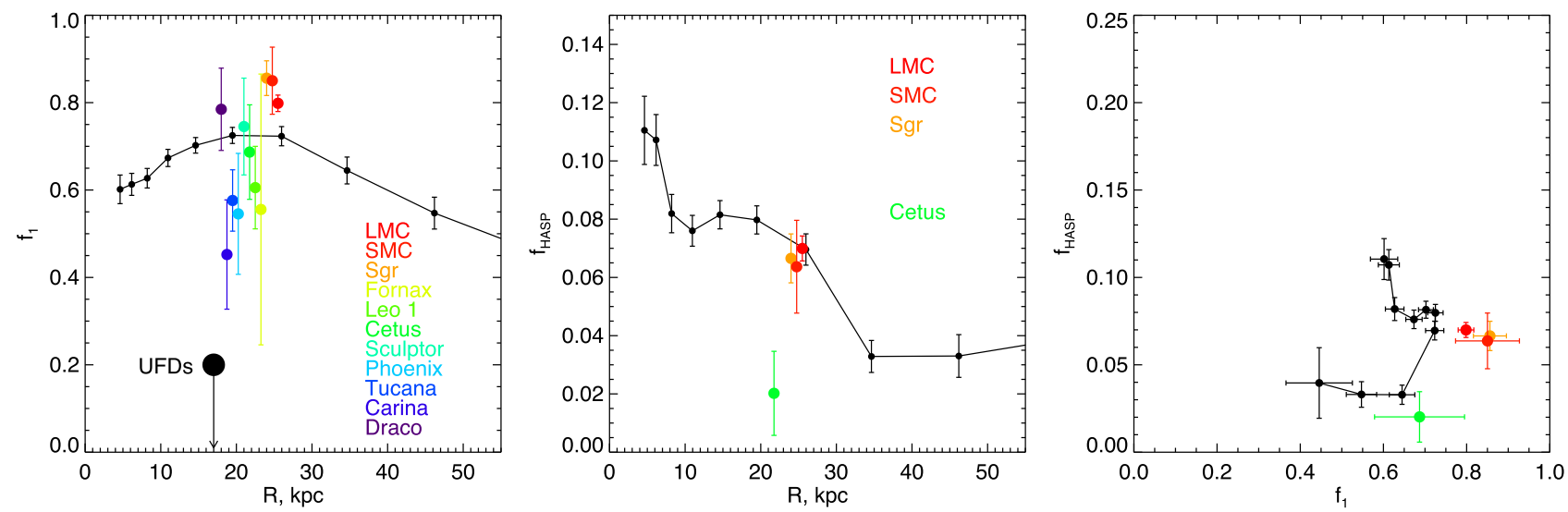

Figure 5. Same as Fig. 4 but for surviving Galactic dSphs. Note that the colour coding is different here, with colour representing the satellite's stellar mass. Additionally, the dSphs are not shown at their current distances in the Galaxy, but are instead offset to the fiducial $R \sim 20 \mathrm{kpc}$ for comparison with the stellar halo measurement (solid black). For the UFDs, we show a tentative measurement of $f_{1}$ based on the data for UMa I (0.2), CVn I (0.23), and Boo I (0.0). For the vast majority of other (fainter) UFDs in the Galaxy, the number of RRab stars is too low to warrant a reliable measurement of the class fraction. Note, however, that the bulk of the RR Lyrae stars in the low-luminosity UFDs are likely Type 2, thus indicating $f_{1} \sim 0$ for the population of the UFDs as a whole. A correlation between $f_{1}$ and dwarf's stellar mass is visible in the left-hand panel. While the surviving dwarfs - occupying the so-called OoInt regime - match well the range of the stellar halo's $f_{1}$, none of the currently observable systems reach the high levels of $f_{\text {HASP }}$ reported for the field halo.

of the (fainter) UFDs (due to small numbers of RR Lyrae detected), it is likely that the ultrafaint ensemble as a whole has $f_{1}$ very close to zero (as indicated by the black arrow).

In terms of the HASP population (middle panel), none of the dwarfs can attain $f_{\text {HASP }} \sim 0.1$ observed near the Galactic Centre. In fact, only three satellites, namely Sgr, the SMC, and the LMC have enough HASP stars to warrant a believable measurement. Curiously, in terms of both $f_{1}$ and $f_{\text {HASP }}$ values, the Sgr dwarf appears to sit around the top of the distribution, thus indirectly confirming that the progenitor system was one of the most massive satellites of the MW, in accord with the studies of Niederste-Ostholt et al. (2010) and Gibbons, Belokurov \& Evans (2017). Finally, as the right-hand panel of Fig. 5 illustrates, the values of $f_{1}$ in the top three most massive dwarfs around the MW are too high, while, at the same time, $f_{\text {HASP }}$ fractions are too low in comparison to the field halo. These results are in good agreement with the earlier measurements by Stetson et al. (2014) and Fiorentino et al. (2015).

\section{HALO SUBSTRUCTURE}

Fig. 6 compares density distributions of Type 1 and two RR Lyrae at three different heliocentric distances. At each distance, the halo looks remarkably different depending on the RRab type used. The choice between Types 1 and 2 affects the appearance of both the smooth component and the substructure. The largest streams such as Sgr (see e.g. Majewski et al. 2003; Belokurov et al. 2006) and VSS (see Duffau et al. 2006, 2014; Newberg et al. 2007) are seen predominantly in the left-hand column, while the narrower/colder Orphan (Grillmair 2006; Belokurov et al. 2007) is clearly discernible only on the right. Moreover, splitting the halo RRab population according to the position in the Bailey diagrams helps to clarify the substructure's $3 \mathrm{D}$ behaviour. For example, the Sgr trailing stream separates cleanly into the faint and the bright components (see Koposov et al. 2012) when Type 1 objects are considered. As evidenced by the middle left- and bottom left-hand panels of the figure, these two branches are not only off-set on the sky but also are located at slightly different distances, in agreement with previous measurements (see Slater et al. 2013).

\subsection{Stellar streams}

To further study the mixture of RR Lyrae stars in previously identified halo substructures, we select likely members of the three stellar streams: Sgr, VSS, and Orphan. Additionally, we split the Sgr stream into four portions: the trailing and leading tails are each divided into a 'bright' and 'faint' component following the classification introduced in Belokurov et al. (2006) and Koposov et al. (2012) that relies on the stream latitude $|B|$. The RA and Dec. selection boundaries for each stream are shown in the top left-hand panel of Fig. 7. These regions are chosen to follow the great circles that approximately match the average stream track on the sky. We aid the 2D selection with a cut on heliocentric distance as illustrated in the bottom left-hand panel of the figure. The resulting measurements of $f_{1}\left(f_{\text {HASP }}\right)$ fractions are given in the middle (right) panel of the figure. Note that, even though the selection is performed in $3 \mathrm{D}$, the samples of likely stream members do suffer from field halo contamination. Thus the type fraction estimates are biased towards the typical field value at the corresponding position, in contrast with the measurements in the Galactic satellites where the contamination is essentially negligible.

As the middle and the right-hand panels of Fig. 7 demonstrate, even in the presence of some field contamination, a diversity of RRab mixtures is observed in the Galactic stellar streams. The Sgr stream and the VSS possess the highest values of $f_{1}$. For Sgr, this is another indication of the high original mass and the relatively fast enrichment history of the progenitor galaxy (see also de Boer et al. 2014; de Boer, Belokurov \& Koposov 2015; Gibbons, Belokurov \& Evans 2017). As analysed here, the leading debris is further away from the progenitor compared to the trailing material, and, accordingly, is dynamically older, i.e. stripped earlier. Thus, the difference in the RRab mixture between the leading and trailing tails - if detected at an appropriate significance level - could strengthen our understanding of the metallicity gradients in the Sgr dwarf before in-fall. Note that population gradients along the stream have been detected previously (see Monaco et al. 2007; Chou et al. 2010; Hyde et al. 2015). These spectroscopic measurements agree with a strong decrease in both $f_{1}$ and $f_{\text {HASP }}$ between the progenitor and the stream as measured here. Note that the RR Lyrae have been used 

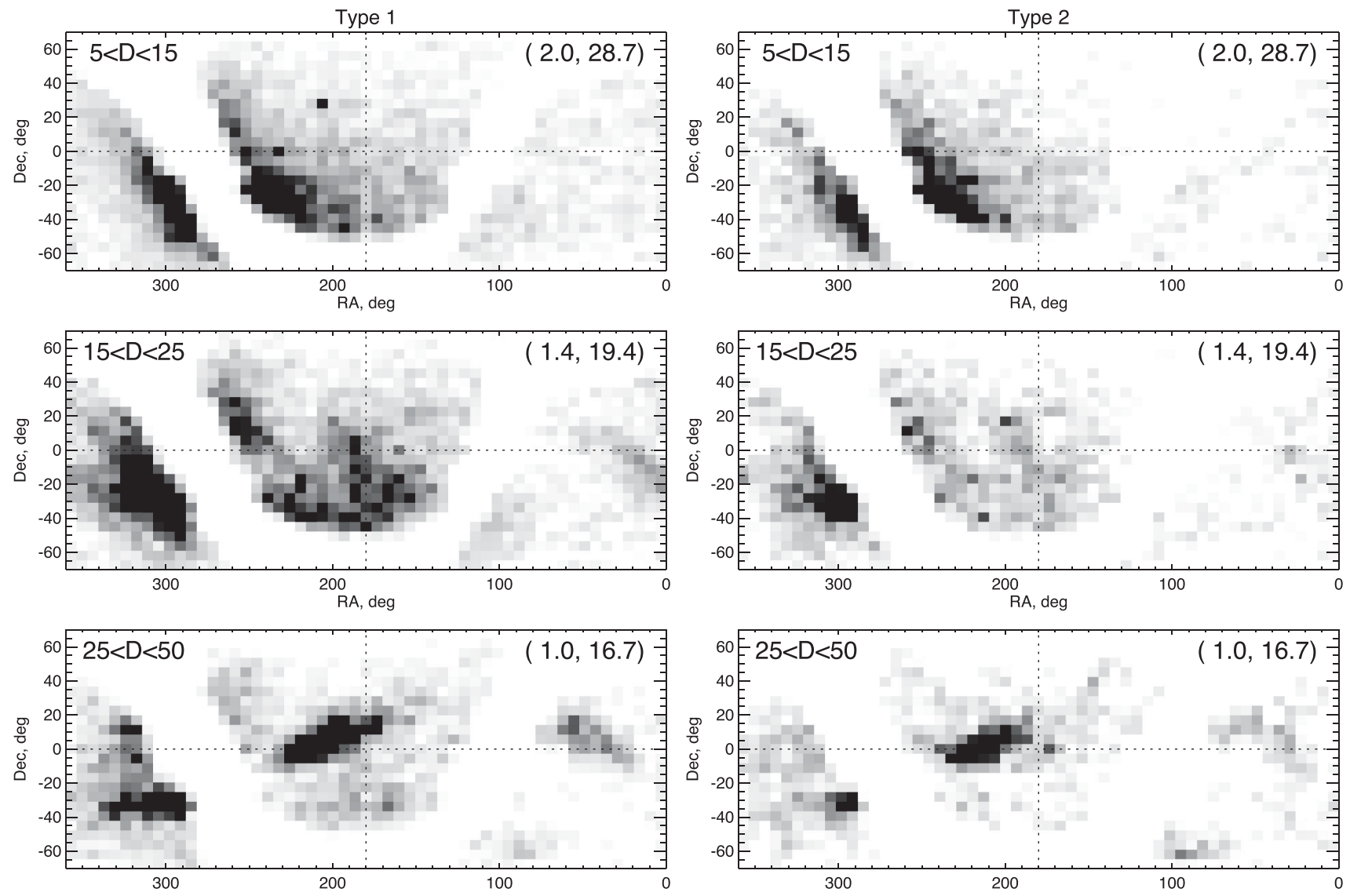

Figure 6. Density of RRab stars of Type 1 (left) and Type 2 (right) in equatorial coordinates for three heliocentric distance range (shown in the top left-hand corner of each panel). There are 55 pixels in the RA dimension and 25 along Dec. The maps are smoothed with a Gaussian kernel with FWHM of 1.1 pixels. The number of stars per pixel corresponding to the black and white shades of grey is shown in the top right-hand corner of each panel. The 'hot' overdense pixels in the density maps correspond to the known satellites of the Galaxy, mostly GCs.
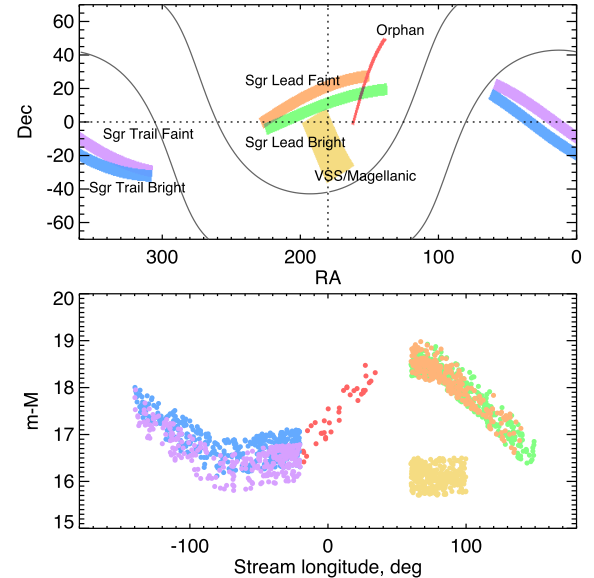
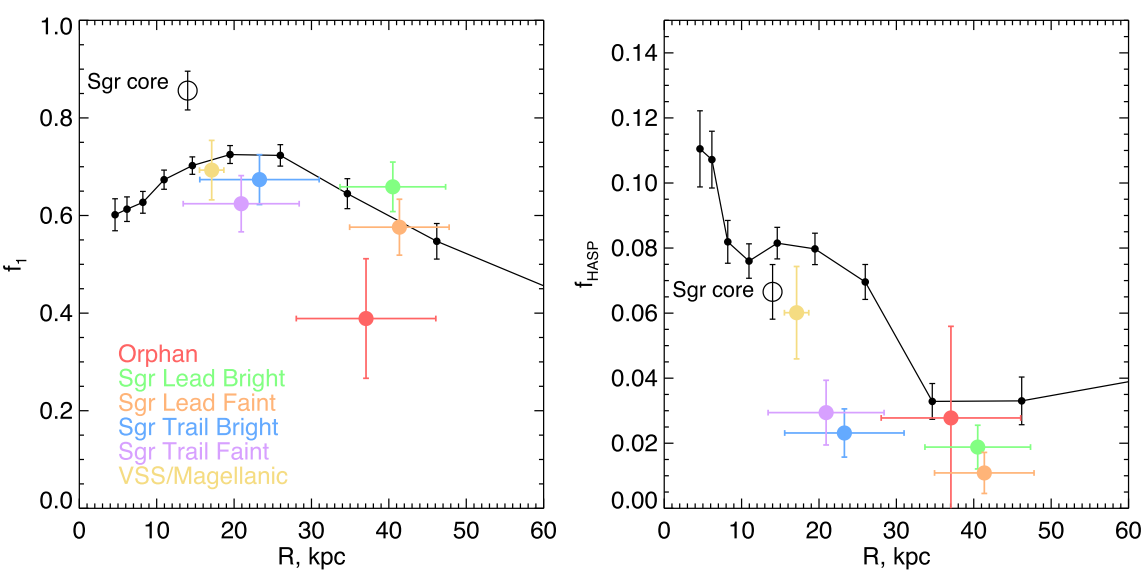

Figure 7. Top left: selection boundaries in equatorial coordinates for each stellar stream considered in this paper. Bottom left: stream candidate member stars in the plane of stream longitude and heliocentric distance. Middle: fraction $f_{1}$ computed for stream candidate member stars (selected as shown on the left) compared to the field stellar halo (black solid). Right: $f_{\text {HASP }}$ for the selected streams. Empty black circle shows the measurement of the RRab class fraction for the Sgr dwarf core (see Fig. 5).

before to trace metallicity gradients in dwarf satellites of the MW (see e.g. Martínez-Vázquez et al. 2016). Moreover, the difference in $f_{1}$ between the bright and faint branches, if significant, could help in the future to shed light on to the creation of the so-called stream bifurcation (see Belokurov et al. 2006; Koposov et al. 2012).
Currently, three models have been put forward to explain the split in the Sgr tails (Fellhauer et al. 2006; Peñarrubia et al. 2010; Gibbons et al. 2016). In all three scenarios, the dynamical age of the debris in the two branches is different, which can be exploited to link back to the stellar populations gradients in the progenitor before the in-fall. 
For the VSS, the elevated fractions $f_{1}$ and $f_{\text {HASP }}$ may signify much higher levels of contamination, which is not surprising given that this is the 'fluffiest' structure of the ones considered here. Alternatively, this could be a clue that the VSS originated in a massive accretion event. For example, Boubert et al. (2017) have recently extended the view of the stream further below the celestial equator and pointed out that the VSS is perfectly aligned with the Magellanic Stream (MS), thus speculating that the structure is nothing else but the leading arm of the disrupting MCs. Note that in all simulations of the MS, such a stream is produced by stripping the SMC rather than the LMC. Therefore, to test the hypothesis of the VSS origin, one ought to compare the stellar populations in the stream to those reported for the SMC. According to the studies of Duffau et al. (2006, 2014), the mean metallicity of the RR Lyrae in the VSS is $[\mathrm{Fe} / \mathrm{H}] \sim-1.8$, which agrees well with the measurement of $[\mathrm{Fe} / \mathrm{H}]=-1.7$ for the RR Lyrae in the SMC (see Haschke et al. 2012). Intriguingly, a stream-like alignment of several GCs coincident with the VSS was reported by Yoon \& Lee (2002). However, unlike the stellar component of the VSS, the GCs in the alleged stream represent a metal-poor subgroup of the OoII objects.

Of the streams considered here, Orphan stands out as the only structure likely dominated by the Class 2 RR Lyrae. The $f_{1} \sim 0.4$ in the stream is at the lowest limit of the range of the values displayed by the classical dwarfs. However, the stream's Class 1 fraction measured here is most likely an upper bound, given the considerable contamination by the field halo. Therefore, we propose that the progenitor of the Orphan stream was a UFD, in contrast with the conclusion reached in the recent study of Hendel et al. (2017).

\section{DISCUSSION AND CONCLUSIONS}

This paper demonstrates that in the MW field halo, there exists a strong evolution of the mixture of RRab stars as a function of Galactocentric radius. More precisely, the fraction of Type 1 (approximately corresponding to $\mathrm{OoI}$ ) stars changes from 60 per cent in the inner $10-15 \mathrm{kpc}$ to 75 per cent at $25 \mathrm{kpc}$, falling back to 60 per cent beyond $40 \mathrm{kpc}$. There is a remarkable agreement between the location of the bump in the $f_{1}$ fraction and the break in the radial density of the stellar halo (see Watkins et al. 2009; Deason et al. 2011; Sesar et al. 2011). As such, our results are in direct contradiction with those presented in Zinn et al. (2014), but we attribute the disagreement to the differences in the size of RR Lyrae samples used. The $f_{1}$ behaviour can be compared to the change in the fraction of HASP RRab variables. The $f_{\text {HASP }}$ ratio also shows an increase at around $\sim 20 \mathrm{kpc}$ and an even steeper fall-off beyond $30 \mathrm{kpc}$, where the HASP fraction drops by a factor of 5. Unlike the Type 1 RRab profile, however, there appears to be an excess of HASP RR Lyrae close to the Galactic Centre (i.e. within the innermost $10 \mathrm{kpc}$ or so). Using simple toy models of the stellar halo, we show that the contribution from at least three different accretion components is required to explain the patterns in the $f_{1}$ and $f_{\text {HASP }}$ fractions. As we elucidate in Section 3, one plausible interpretation of the peak in both $f_{1}$ and $f_{\text {HASP }}$ fractions at $\sim 25 \mathrm{kpc}$ is a combination of (i) different flattening of the spatial distribution of shorter period RR Lyrae stars and (ii) a sharp truncation of the distribution of the RR Lyrae with short periods. Both of these conditions may be accommodated in a scenario with an early accretion of a single massive system (see Deason et al. 2013).

To help calibrate the $f_{1}$ and $f_{\text {HASP }}$ estimates, we also analyse the mixture of RRab stars in the surviving MW satellites, such as GCs and dSphs (see Figs 4 and 5). As noted previously by many authors, the GCs show a clear dichotomy, with some preferring low values of $f_{1}$ and some high. The dSphs, on the other hand, tend to have intermediate values of $f_{1}$. While many of the GCs lie either too low or too high compared to the $f_{1}$ in the field halo, dSphs appear to match the range of the observed halo Type 1 fraction much better. This does not necessarily imply that the halo field (or even individual $\mathrm{dSphs}$, for that matter) is devoid of the Oosterhoff dichotomy, as intermediate $f_{1}$ values between those seen in OoI and OoII objects could in principle also arise from an arbitrary mix containing these two types of stars. We register a correlation between the dwarf's stellar mass and $f_{1}$, with the three most massive satellites, namely Sgr, the SMC, and the LMC, reaching $f_{1} \sim 80$ per cent, slightly above the halo peak of $f_{1} \sim 75$ percent. Even though there exist objects amongst both the GCs and the dSphs with sufficiently high $f_{1}$ values, neither of the two satellite classes contains fractions of HASP RR Lyrae similar to the halo. The bulk of the GCs and the $\mathrm{dSphs}$ (including the most massive ones) contain a factor of 2 lower numbers of HASP variables. There is a small number of GCs with fractions $f_{\text {HASP }}$ a factor of 1.5 higher compared to the peak of the field halo. However, as the right-hand panel of Fig. 4 illustrates, the exact combination of intermediate $f_{1}$ and elevated $f_{\text {HASP }}$ is not realized in any of the surviving satellites, be it a GC or a dSph.

Stepping aside from studying the average properties of the RR Lyrae mixture in the field, maps of the RRab density shown in Fig. 6 reveal striking differences in the properties of the stellar halo depending on the type of the pulsator used. We choose three wellknown halo substructures whose 3D properties have been mapped out previously, namely the Sgr stream, the VSS, and the Orphan stream. We detect clear signs of evolution of both $f_{1}$ and $f_{\text {HASP }}$ along the Sgr tails: compared to the progenitor, the stream's $f_{1}$ is reduced by 30 per cent and $f_{\text {HASP }}$ by a factor of 3 . We hypothesize that our measurements are consistent with chemical abundance gradients in the progenitor. We suggest that in the future, a more accurate RR Lyrae fraction estimate might help elucidate the models of the Sgr dwarf disruption and the genesis of the stream bifurcation. Surprisingly, we find that the VSS possesses high values of both $f_{1}$ and $f_{\text {HASP }}$, which would imply its origin in a massive galaxy, perhaps similar to the SMC. If not a result of field halo contamination, our measurement lends support to the recent discovery of Boubert et al. (2017), who point out a close connection between the extended view of the VSS they uncover and the Magellanic Clouds. Finally, the Orphan stream contains the lowest fraction of Type 1 RR Lyrae, $f_{1} \sim 0.4$, which, given a likely non-zero contamination, is only an upper bound on its true $f_{1}$. Our measurement of $f_{1}$ for the Orphan stream has two important consequences. First, this value is smaller than those for the classical dSphs, and may be in better agreement with the extremely low values of $f_{1}<0.2$ for the surviving UFDs. Second, it shows that the search for low-mass substructure in the halo can be significantly improved by using a particular subset of RRab stars, more precisely those belonging to Class 2 .

What is the most straightforward interpretation of the radial evolution of the $f_{1}$ fraction as shown in Fig. 2? Given the correlation between the stellar mass and the $f_{1}$ fraction exhibited by the dSphs currently in orbit around the $\mathrm{MW}$, the Galactic $f_{1}$ profile may simply be a reflection of the change in the fractional contribution of the halo progenitors of different masses. In other words, at distances below $15 \mathrm{kpc}$ and beyond $40 \mathrm{kpc}$ where $f_{1}$ is at its lowest, an enhanced contribution from lower mass systems is expected. Between 15 and $30 \mathrm{kpc}$, where the peaks in both $f_{1}$ and $f_{\text {HASP }}$ are observed, the stellar halo is dominated by the debris from a massive progenitor - a hypothesis similar to the halo break theory put forward by Deason et al. (2013). To test this conjecture, we analyse a suite of cosmo- 

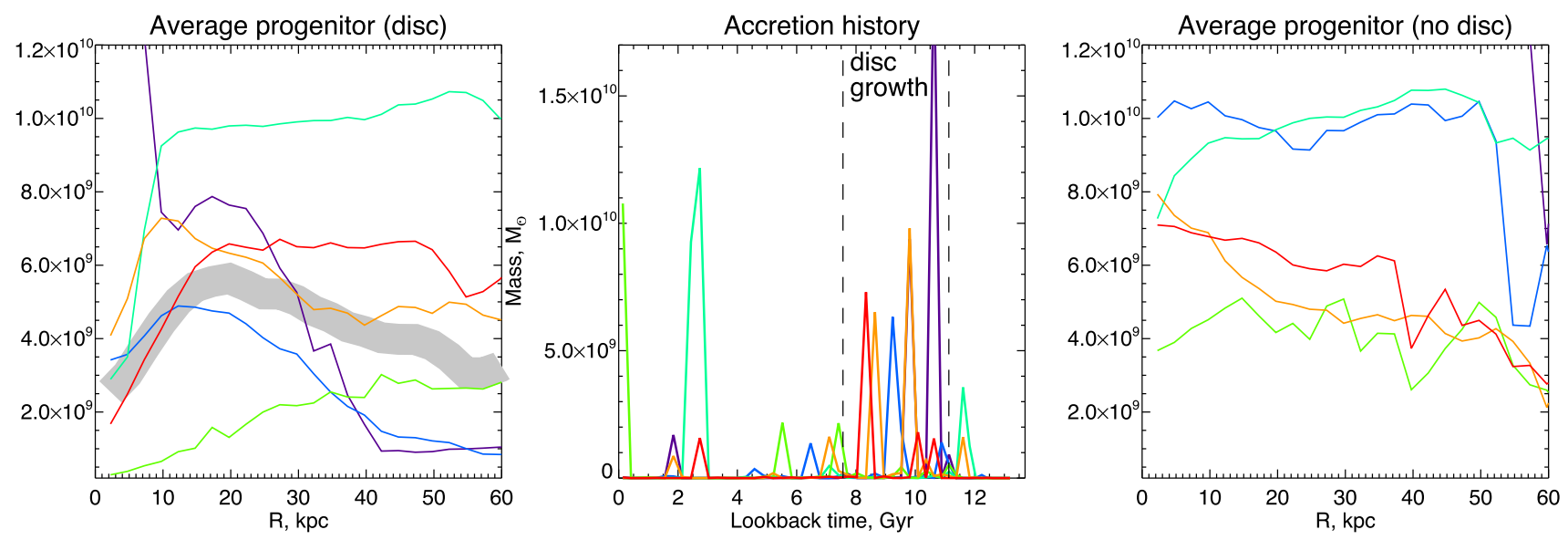

Figure 8. Left: mean progenitor mass at the given Galactocentric distance in six simulated MW stellar haloes. The thick grey band represents the average between the blue and the red curves. Colour is used to distinguish between individual haloes and link to the accretion history shown in the middle panel. Middle: accretion histories of the simulated stellar haloes. Right: same as left-hand panel, but for stellar haloes simulated without a baryonic disc. Note that the disc grown between redshifts 3 and 1 affects the debris distribution in the stellar halo and can sometimes turn a rather flat progenitor mass profile (as shown in the right-hand panel) into a 'bumpy' one (as shown in the left-hand panel).

logical zoom-in simulations of MW halo formation. The details of the simulations can be found in Jethwa, Belokurov \& Erkal (2018). In essence, the six simulated stellar haloes considered here sample a range of MW masses and capture some of the effects induced by the baryons present in the real Galaxy, i.e. the action of the disc. The baryonic disc is implemented parametrically and is grown adiabatically between redshifts 3 and 1. To create the stellar halo, the 3 percent most bound dark matter particles in each in-falling subhalo are tagged as stars. This is done by determining when each subhalo reaches its peak mass and then assigning it a stellar mass of

$M_{*}=3.4 \times 10^{6} \mathrm{M}_{\odot}\left(\frac{M_{\text {peak }}}{10^{9} \mathrm{M}_{\odot}}\right)^{1.7}$,

similar to the approach in De Lucia \& Helmi (2008) and Bailin et al. (2014). In order to only sample the stellar halo, stars within 2 tidal radii of the remaining subhaloes at $z=0$ are excluded.

The left-hand panel of Fig. 8 presents the average progenitor stellar mass as a function of Galactocentric radius in the six simulated haloes whose accretion histories are displayed in the middle panel. All (but one) haloes display a drop in the average progenitor mass at small distances from the halo's centre. The fake MWs with extended or recent accretion (shades of green) display a flat mass profile across the range of distances considered here, i.e. between 20 and $70 \mathrm{kpc}$. Those haloes whose accretion history peaked early, i.e. those coloured purple, blue, orange, and red all show a drop in the average progenitor mass beyond $30-60 \mathrm{kpc}$. Clearly, the exact shape of the mean progenitor mass profile is very sensitive to the details of the host accretion history, which cannot be exhaustively sampled with the small number of simulations presented here. For illustration purposes, we also show a combination of the two mean mass profiles (for the haloes shown in red and blue) as a thick grey line. As demonstrated in the figure, MWs with an accretion history peaking between 8 and $10 \mathrm{Gyr}$ ago can indeed possess a stellar halo with a characteristic bump in the mean progenitor mass profile, i.e. very similar to the one implied by the RRab studies presented here. Curiously, as shown in the right-hand panel of Fig. 8, the corresponding zoom-in simulations without a disc do not show any of the trends discussed above. Regardless of the accretion history, between 5 and $50 \mathrm{kpc}$, the mean progenitor mass profiles appear rather flat. We therefore conclude that the changes in the mean mass profile as seen in the left-hand panel of the figure are put in place by the action of the disc. As the disc grows from $\sim 11$ to $\sim 8 \mathrm{Gyr}$ ago, it helps to migrate the stellar debris accumulated so far from the outer parts of the halo into the inner regions. Around the Galactic enter, this 'debris sorting' induced by the disc growth creates an excess of the material accreted the earliest (11-13 Gyr) - and thus mostly contributed by the lower mass objects (as bigger dwarfs require some time to grow before falling into the MW). The stars stripped from the larger systems later on $(8-11 \mathrm{Gyr})$ are moved closer to the Galactic Centre but cannot be packed as tightly, hence resulting in an overdensity at intermediate radii (20-30 kpc). Therefore, the three stellar halo components required by the RR Lyrae mixture changes could be (i) the debris from low-mass objects accreted early (10-13 Gyr) and contributing to the inner region of the halo today ( $<15 \mathrm{kpc}$ ); (ii) material from a massive system merging with the MW between 8 and 11 Gyr ago and dominating the halo at the distances between 10 and $30 \mathrm{kpc}$; and (iii) the stars from the rest of the unlucky Galactic satellites fallen into the MW during its lifetime.

To summarize, the changes in the RRab mixture with radius can be explained with the evolution of the relative contribution of progenitors of different masses. Given the behaviour of $f_{1}$, the field halo at low and high Galactocentric distances might be dominated by debris from systems not unlike a typical dSph observed around the MW today. The intermediate distances in the Galaxy appear to have an increased contribution from the most massive systems, perhaps as massive as Sgr/SMC/LMC. However, the exact nature of such a 'giant dwarf' progenitor is unclear as the halo's $f_{\text {HASP }}$ fraction at these distances is almost twice that of the most massive surviving dwarf (LMC). Of course, such an ancient progenitor system might not necessarily be a replica of the LMC. Note that the LMC which appears to have arrived to the Galaxy rather recently (see e.g. Kallivayalil et al. 2013) - has had a prolonged period of relative inactivity, restarting star formation in earnest only $5 \mathrm{Gyr}$ ago (see Harris \& Zaritsky 2009; Meschin et al. 2014; Monteagudo et al. 2018). The hypothesized halo progenitor, on the other hand, evolved much closer to the MW, and thus likely had a different - probably faster - enrichment history. If a large portion of Type 1 RR Lyrae stars around the $15-30 \mathrm{kpc}$ has indeed been contributed via an early merger with a massive system, it may be possible to pick up tracers 
of this accretion event in the kinematics of stars in the future Gaia data releases. Note that many OoI GCs tend to prefer retrograde orbits according to van den Bergh (1993a,b), who suggested the merger of a massive ancestral object(s) on a retrograde orbit as the origin of the MW's OoI component. With regards to the shortperiod RRab stars, it appears rather plausible that the sharp rise in $f_{\text {HASP }}$ as one approaches the Galactic Centre is due to an increasing contribution by bulge stars, in agreement with the earlier studies of Kunder \& Chaboyer (2009). Finally, our hypothesis of a strong evolution in the mass of the 'typical' contributing progenitor as a function of Galactocentric radius appears to be supported by a comparison to the cosmological zoom-in simulations of the MW halo formation in the presence of a baryonic disc.

The ideas discussed here are complementary to the analysis of the stellar population mixture as encoded in colour-magnitude space (see e.g. de Jong et al. 2010) and have many similarities with other studies of variations in the ratio of distinct halo tracers (see Bell et al. 2010; Deason et al. 2015; Price-Whelan et al. 2015). Given the diversity of the behaviour of the simple test statistics proposed here, such as $f_{1}$ and $f_{\text {HASP }}$ fractions, we surmise that the distribution of RR Lyrae in the period-amplitude space appears to be unique enough to be used as a progenitor fingerprint. In particular, it may be possible to use it to tease out signatures of the accretion of low-mass satellites. There is also an added value in being able to leverage the unique properties of the RR Lyrae as standard candles to disentangle the hotchpotch of the stellar halo in 3D. All in all, in the view of the imminent Gaia Data Release 2, unmixing the Galactic halo with RR Lyrae tagging should become feasible in the near future.

\section{ACKNOWLEDGEMENTS}

The authors wish to thank Kathryn Johnston and Douglas Boubert for stimulating discussions that helped to improve this paper. VB is grateful to Natàlia Mora-Sitjà for the careful proofreading of the manuscript. The authors thank the anonymous referee for thorough reading of the paper.

The research leading to these results has received funding from the European Research Council under the European Union's Seventh Framework Programme (FP/2007-2013)/ERC Grant Agreement no. 308024. AJDe is supported by a Royal Society University Research Fellowship. AJDe also acknowledges support from the STFC grant ST/P000451/1. NWE thanks the Center for Computational Astrophysics for hospitality during a working visit.

The authors gratefully acknowledge support by CONICYT/RCUK's PCI program through grant DPI20140066 ('Newton Funds'). MC is additionally supported by the Ministry for the Economy, Development, and Tourism's Millennium Science Initiative through grant IC 120009, awarded to the Millennium Institute of Astrophysics (MAS); by Proyecto Basal PFB-06/2007; and by FONDECYT grant \#1171273.

\section{REFERENCES}

Abbas M. A., Grebel E. K., Martin N. F., Burgett W. S., Flewelling H., Wainscoat R. J., 2014, MNRAS, 441, 1230

Allende Prieto C. et al., 2008, Astron. Nachr., 329, 1018

Arp H. C., 1955, AJ, 60, 317

Bailin J., Bell E. F., Valluri M., Stinson G. S., Debattista V. P., Couchman H. M. P., Wadsley J., 2014, ApJ, 783, 95

Battaglia G., North P., Jablonka P., Shetrone M., Minniti D., Díaz M., Starkenburg E., Savoy M., 2017, A\&A, 608, A145
Bekki K., Tsujimoto T., 2016, ApJ, 831, 70

Bell E. F., Xue X. X., Rix H.-W., Ruhland C., Hogg D. W., 2010, AJ, 140, 1850

Belokurov V. et al., 2006, ApJ, 642, L137

Belokurov V. et al., 2007, ApJ, 658, 337

Bensby T., Feltzing S., Oey M. S., 2014, A\&A, 562, A71

Bernard E. J. et al., 2009, ApJ, 699, 1742

Blanco-Cuaresma S. et al., 2015, A\&A, 577, A47

Bland-Hawthorn J., Krumholz M. R., Freeman K., 2010, ApJ, 713, 166

Bono G., Caputo F., Castellani V., Marconi M., 1997, A\&AS, 121, 327

Boubert D., Belokurov V., Erkal D., Iorio G., 2017, preprint (arXiv:1707.03833)

Bovy J., 2016, ApJ, 817, 49

Boylan-Kolchin M., 2017, MNRAS, 472, 3120

Carollo D. et al., 2007, Nature, 450, 1020

Carollo D. et al., 2010, ApJ, 712, 692

Carretta E., Bragaglia A., Gratton R. G., Recio-Blanco A., Lucatello S., D’Orazi V., Cassisi S., 2010, A\&A, 516, A55

Castellani V., 1983, Mem. Soc. Astron. Ital., 54, 141

Catelan M., 2004, in Kurtz D. W., Pollard K. R., eds, ASP Conf. Ser. Vol. 310, IAU Colloq. 193, Variable Stars in the Local Group. Astron. Soc. Pac., San Francisco, p. 113

Catelan M., 2009, Ap\&SS, 320, 261

Chou M.-Y., Cunha K., Majewski S. R., Smith V. V., Patterson R. J., Martínez-Delgado D., Geisler D., 2010, ApJ, 708, 1290

Chung C., Lee Y.-W., Pasquato M., 2016, MNRAS, 456, L1

Clement C., 2017a, in Catelan M., Gieren W., eds, EPJ Web Conf. Vol. 152, Wide-Field Variability Surveys: A 21st Century Perspective. EDP Sciences, Les Ulis, France, p. 01021

Clement C. M., 2017b, VizieR Online Data Catalog, 5150

Clement C. M. et al., 2001, AJ, 122, 2587

Clementini G., 2014, in Guzik J. A., Chaplin W. J., Handler G., Pigulski A., eds, Proc. IAU Symp. Vol. 301, Precision Asteroseismology. Cambridge Univ. Press, Cambridge, p. 129

Coppola G. et al., 2013, ApJ, 775, 6

Cui X.-Q. et al., 2012, RA\&A, 12, 1197

Dall'Ora M. et al., 2006, ApJ, 653, L109

Das P., Williams A., Binney J., 2016, MNRAS, 463, 3169

Deason A. J., Belokurov V., Evans N. W., 2011, MNRAS, 416, 2903

Deason A. J., Belokurov V., Evans N. W., Johnston K. V., 2013, ApJ, 763, 113

Deason A. J., Belokurov V., Weisz D. R., 2015, MNRAS, 448, L77

Deason A. J., Belokurov V., Koposov S. E., Gómez F. A., Grand R. J., Marinacci F., Pakmor R., 2017, MNRAS, 470, 1259

de Boer T. J. L., Belokurov V., Beers T. C., Lee Y. S., 2014, MNRAS, 443, 658

de Boer T. J. L., Belokurov V., Koposov S., 2015, MNRAS, 451, 3489

de Jong J. T. A., Yanny B., Rix H.-W., Dolphin A. E., Martin N. F., Beers T. C., 2010, ApJ, 714, 663

De Lucia G., Helmi A., 2008, MNRAS, 391, 14

De Silva G. M., Freeman K. C., Asplund M., Bland-Hawthorn J., Bessell M. S., Collet R., 2007, AJ, 133, 1161

De Silva G. M. et al., 2015, MNRAS, 449, 2604

Dotter A., 2008, ApJ, 687, L21

Drake A. J. et al., 2013a, ApJ, 763, 32

Drake A. J. et al., 2013b, ApJ, 765, 154

Drake A. J. et al., 2014, ApJS, 213, 9

Drake A. J. et al., 2017, MNRAS, 469, 3688

Duffau S., Zinn R., Vivas A. K., Carraro G., Méndez R. A., Winnick R., Gallart C., 2006, ApJ, 636, L97

Duffau S., Vivas A. K., Zinn R., Méndez R. A., Ruiz M. T., 2014, A\&A, 566, A118

Fellhauer M. et al., 2006, ApJ, 651, 167

Fiorentino G. et al., 2015, ApJ, 798, L12

Font A. S., Johnston K. V., Bullock J. S., Robertson B. E., 2006, ApJ, 638 , 585

Freeman K., Bland-Hawthorn J., 2002, ARA\&A, 40, 487

Garofalo A. et al., 2013, ApJ, 767, 62 
Gibbons S. L. J., Belokurov V., Erkal D., Evans N. W., 2016, MNRAS, 458, L64

Gibbons S. L. J., Belokurov V., Evans N. W., 2017, MNRAS, 464, 794

Gilmore G. et al., 2012, The Messenger, 147, 25

Gratton R. G., Carretta E., Bragaglia A., Lucatello S., D’Orazi V., 2010, A\&A, 517, A81

Gratton R. G., Carretta E., Bragaglia A., 2012, A\&AR, 20, 50

Greco C. et al., 2009, ApJ, 701, 1323

Grillmair C. J., 2006, ApJ, 645, L37

Harris J., Zaritsky D., 2009, AJ, 138, 1243

Haschke R., Grebel E. K., Duffau S., Jin S., 2012, AJ, 143, 48

Helmi A., Navarro J. F., Nordström B., Holmberg J., Abadi M. G., Steinmetz M., 2006, MNRAS, 365, 1309

Hendel D. et al., 2017, preprint (arXiv:1711.04663)

Hyde E. A. et al., 2015, ApJ, 805, 189

Iorio G., Belokurov V., Erkal D., Koposov S. E., Nipoti C., Fraternali F., 2018, MNRAS, 474, 2142

Jang S., Lee Y.-W., 2015, ApJS, 218, 31

Jang S., Lee Y.-W., Joo S.-J., Na C., 2014, MNRAS, 443, L15

Jethwa P., Erkal D., Belokurov V., 2018, MNRAS, 473, 2060

Kallivayalil N., van der Marel R. P., Besla G., Anderson J., Alcock C., 2013, ApJ, 764, 161

Kaluzny J., Kubiak M., Szymanski M., Udalski A., Krzeminski W., Mateo M., 1995, A\&AS, 112, 407

Kapakos E., Hatzidimitriou D., Soszyński I., 2011, MNRAS, 415, 1366

Kinemuchi K., Harris H. C., Smith H. A., Silbermann N. A., Snyder L. A., La Cluyzé A. P., Clark C. L., 2008, AJ, 136, 1921

Koposov S. E. et al., 2012, ApJ, 750, 80

Kruijssen J. M. D., 2015, MNRAS, 454, 1658

Kuehn C. et al., 2008, ApJ, 674, L81

Kunder A., Chaboyer B., 2009, AJ, 138, 1284

Kunder A., Stetson P. B., Catelan M., Walker A. R., Amigo P., 2013, AJ, 145,33

Lee J.-W., Carney B. W., 1999, AJ, 118, 1373

Lee Y.-W., Demarque P., Zinn R., 1990, ApJ, 350, 155

Majewski S. R., Skrutskie M. F., Weinberg M. D., Ostheimer J. C., 2003, ApJ, 599, 1082

Majewski S. R., Nidever D. L., Smith V. V., Damke G. J., Kunkel W. E., Patterson R. J., Bizyaev D., García Pérez A. E., 2012, ApJ, 747, L37

Majewski S. R. et al., 2017, AJ, 154, 94

Martell S. L., Grebel E. K., 2010, A\&A, 519, A14

Martell S. L., Smolinski J. P., Beers T. C., Grebel E. K., 2011, A\&A, 534, A136

Martínez-Vázquez C. E. et al., 2016, MNRAS, 461, L41

Meschin I., Gallart C., Aparicio A., Hidalgo S. L., Monelli M., Stetson P. B., Carrera R., 2014, MNRAS, 438, 1067

Miceli A. et al., 2008, ApJ, 678, 865

Minchev I., Chiappini C., Martig M., 2013, A\&A, 558, A9

Minchev I., Chiappini C., Martig M., 2014, A\&A, 572, A92

Mitschang A. W., De Silva G., Zucker D. B., Anguiano B., Bensby T., Feltzing S., 2014, MNRAS, 438, 2753

Monaco L., Bellazzini M., Bonifacio P., Buzzoni A., Ferraro F. R., Marconi G., Sbordone L., Zaggia S., 2007, A\&A, 464, 201
Monteagudo L., Gallart C., Monelli M., Bernard E. J., Stetson P. B., 2018, MNRAS, 473, L16

Ness M. et al., 2018, ApJ, 853, 198

Newberg H. J., Yanny B., Cole N., Beers T. C., Re Fiorentin P., Schneider D. P., Wilhelm R., 2007, ApJ, 668, 221

Niederste-Ostholt M., Belokurov V., Evans N. W., Peñarrubia J., 2010, ApJ, 712, 516

Oosterhoff P. T., 1939, The Observatory, 62, 104

Oosterhoff P. T., 1944, Bull. Astron. Inst. Netherlands, 10, 55

Peñarrubia J., Belokurov V., Evans N. W., Martínez-Delgado D., Gilmore G., Irwin M., Niederste-Ostholt M., Zucker D. B., 2010, MNRAS, 408, L26

Pietrukowicz P. et al., 2015, ApJ, 811, 113

Preston G. W., 1959, ApJ, 130, 507

Price-Whelan A. M., Johnston K. V., Sheffield A. A., Laporte C. F. P., Sesar B., 2015, MNRAS, 452, 676

Pritzl B. J., Smith H. A., Catelan M., Sweigart A. V., 2002, AJ, 124, 949

Renaud F., Agertz O., Gieles M., 2017, MNRAS, 465, 3622

Renzini A., 1983, Mem. Soc. Astron. Ital., 54, 335

Roškar R., Debattista V. P., Quinn T. R., Stinson G. S., Wadsley J., 2008, ApJ, 684, L79

Schlegel D. J., Finkbeiner D. P., Davis M., 1998, ApJ, 500, 525

Schönrich R., Binney J., 2009, MNRAS, 396, 203

Sesar B., Jurić M., Ivezić Ž., 2011, ApJ, 731, 4

Slater C. T. et al., 2013, ApJ, 762, 6

Sollima A., Cassisi S., Fiorentino G., Gratton R. G., 2014, MNRAS, 444, 1862

Soszynski I. et al., 2003, Acta Astron., 53, 93

Soszyński I. et al., 2014, Acta Astron., 64, 177

Stetson P. B., Fiorentino G., Bono G., Bernard E. J., Monelli M., Iannicola G., Gallart C., Ferraro I., 2014, PASP, 126, 616

Ting Y.-S., Conroy C., Goodman A., 2015, ApJ, 807, 104

Tolstoy E., Hill V., Tosi M., 2009, ARA\&A, 47, 371

Torrealba G. et al., 2015, MNRAS, 446, 2251

van Albada T. S., Baker N., 1973, ApJ, 185, 477

VandenBerg D. A., Denissenkov P. A., Catelan M., 2016, ApJ, 827, 2

van den Bergh S., 1993a, AJ, 105, 971

van den Bergh S., 1993b, MNRAS, 262, 588

van den Bergh S., 1993c, ApJ, 411, 178

van den Bergh S., 2011, PASP, 123, 1044

Venn K. A., Irwin M., Shetrone M. D., Tout C. A., Hill V., Tolstoy E., 2004, AJ, 128, 1177

Vivas A. K. et al., 2016, AJ, 151, 118

Watkins L. L. et al., 2009, MNRAS, 398, 1757

Xue X.-X., Rix H.-W., Ma Z., Morrison H., Bovy J., Sesar B., Janesh W., 2015, ApJ, 809, 144

Yoon S.-J., Lee Y.-W., 2002, Science, 297, 578

Zinn R., Horowitz B., Vivas A. K., Baltay C., Ellman N., Hadjiyska E., Rabinowitz D., Miller L., 2014, ApJ, 781, 22

Zorotovic M. et al., 2010, AJ, 139, 357

This paper has been typeset from a $\mathrm{T}_{\mathrm{E}} \mathrm{X} / \mathrm{L} \mathrm{T} \mathrm{T}_{\mathrm{E}} \mathrm{X}$ file prepared by the author. 